\title{
Atividade de colônias de Cornitermes Cumulans (Isoptera, Nasutitermitinae) sobre estruturas edáficas macro e microagregadas em casa de vegetação
}

\section{Cornitermes Cumulans (Isoptera, Nasutitermitinae) colonies activity in controlled conditions on macro and micro-aggregated soil structures}

\author{
Maria Inês Lopes de Oliveira'; ${ }^{1}$ Norton Pólo Benito ${ }^{2}$; Amabilio José Aires de \\ Camargo $^{3}$; Maria de Fátima Guimarães ${ }^{4}$; Michel Brossard ${ }^{5 *}$
}

\begin{abstract}
Resumo
O objetivo deste trabalho foi avaliar o comportamento de térmitas em estruturas oriundas de um Latossolo Vermelho Amarelo. Ninhos em atividade de Cornitermes cumulans (Isoptera, Nasutitermitinae) foram coletados a campo e mantidos em vasos em casa de vegetação por nove meses. As estruturas utilizadas no experimento foram macroestruturas densas e estrutura granular, ambas amostradas no mesmo solo que as colônias. O experimento foi realizado com duas repetições para cada estrutura, sendo cada repetição composta de um vaso com estrutura, ninho e alimento, e um vaso com a mesmo material e alimentação. As estruturas fabricadas pelos térmitas foram descritas macro e micromorfologicamente e comparadas com amostras coletadas no campo, também se verificou a distribuição das galerias. Os térmitas mantiveram as atividades de forrageamento e construção do ninho até o final do experimento, com algumas restrições. Para os vasos com estruturas densas a atividade desenvolveu-se tanto no vaso onde havia o ninho quanto no vaso com alimentação. Em comparação, para os vasos com estrutura granular as construções foram menos desenvolvidas e localizadas nos volumes associados aos ninhos. Nas estruturas construídas, as partículas de solo eram orientadas, formando uma parede rígida, como observado em feições coletadas no campo. As colônias de C. cumulans exploravam o ambiente de forma aleatória em todas as direções, retrabalhando estruturas densas e reagregando as partículas do solo para construção de galerias. Todas as estruturas densas ofertadas às colônias foram parcialmente ou completamente modificadas, e as concentrações de matéria orgânica das estruturas densas oriundas de horizontes profundos do solo foram incrementadas. Os térmitas retrabalham estruturas deixadas por eles ou outros invertebrados do solo, criando microestrutura e re-agregando o solo.

Palavras-chave: Termitária, estrutura do solo, micromorfologia, porosidade, pedoturbação
\end{abstract}

\footnotetext{
Abstract

The aim of this paper is to present the termite impact on sampled soil structures during a controlled experiment. The structures used were sampled in a red yellow Latosol. The Cornitermes cumulans colonies were sampled in the same site and were maintained in controlled conditions during 9 months.

${ }^{1}$ Doutorada em Agronomia da Universidade Estadual de Londrina, UEL, Londrina, PR. E-mail: minesoliveira@yahoo.com.br

2 Pesquisador da Embrapa Recursos Genéticos e Biotecnologia, Brasília, DF. E-mail: norton@cenargen.embrapa.br

3 Pesquisador da Embrapa Cerrados, Planaltina, DF. E-mail: amabilio@cpac.embrapa.br

${ }^{4}$ Prof $^{\mathrm{a}} \mathrm{Dr}^{\mathrm{a}}$ do Dept $^{\mathrm{o}}$ de Agronomia, UEL, Londrina, PR. E-mail: mfatima@uel.br

5 Pesquisador do Institut de recherche pour le développement, IRD, UMR 210 Eco\&Sols, BP 64501, 34394 Montpellier cedex 5, França. E-mail: michel.brossard@ird.fr

* Autor para correspondência
} 
Two types of structures were tested: dense macrostructures and a strong granular structure sampled in the same site than the colonies. Two replicates of the conceptual termite-structure model were developed. Two pots, one of them with the colony and food material, the second with the same structured material and food, composed each replicate; a tube associated the two pots. Macro and micromorphological descriptions were done, the distribution and localization of galleries were described and compared with those sampled at field. The termites' colonies maintained their exploring and construction activities, during the period, with some restrictions. In the experimental model with dense macrostructures the activities were important and equivalent in all of the volumes. But, in the experimental model with the strong granular structure the activities were essentially done in the volumes with the mounds. The new structures that were built, soil particles were oriented, and formed rigid walls as they were observed in field samples. C. cumulans colonies explored the volume aleatory in all directions. Many of the dense structures offered in the pots to the termite activity were completely or partially modified; the dense structure organic matter concentration were increase in the structures sampled in deep soil horizons. It was clear that the termites transformed structures offered, and that there created new microstructures and aggregates.

Key words: Termitaria, soil structure, micromorphology, porosity, pedoturbation

\section{Introdução}

Os térmitas (Isoptera: Termitidea) criam estruturas biogênicas (galerias, ninhos, câmaras, aterros e outros) que tem um efeito significativo sobre o ambiente em que vivem. As modificações mais visíveis são observadas nos atributos físicos do solo através da seleção, transporte e reorganização das partículas. A importância dos térmitas nas propriedades dos solos está intimamente relacionada com suas atividades de construção e com o tamanho das populações, especialmente na região dos cerrados (FERREIRA; TAVARES FILHO; FERREIRA, 2010, BENITO et al., 2008). O papel dos térmitas na pedoturbação é reconhecido, mas variável segundo os ambientes considerados (OLIVEIRA et al., 2011).

Os térmitas tem influência na estruturação do solo, na decomposição de resíduos vegetais e na disponibilidade de nutrientes, estimulando a atividade microbiana (DEBRUYN; CONACHER, 1995; LAVELLE, 1997). Por meio de suas ações mecânicas modificam a estabilidade de agregados, segundo as condições ecológicas onde se encontram (GARNIER-SILLAM; TOUTAIN; RENOUX, 1988) e podem proteger parte da matéria orgânica de uma mineralização rápida (LAVELLE; SPAIN, 2001; DECÄENS et al., 2003). A modificação dos materiais do solo pelos térmitas se dá pela deposição de compostos orgânicos, saliva e fezes durante a construção dos ninhos (BIGNELL, 2006). Notadamente, os valores de parâmetros químicos, como C, N e P, que são integrados nas estruturas organo-minerais, são em geral, mais elevados nos ninhos que no solo adjacente (BROSSARD et al., 2007; CHAPUIS-LARDY et al., 2011, LÓPEZ-HERNÁNDEZ et al., 2006; LÓPEZHERNÁNDEZ, 2001).

De maneira geral, é reconhecido que a regulação das atividades de construção e escavação de térmitas e formigas é dependente de processos complexos ligados a parâmetros ambientais e físicos (GRASSË, 1959; BONABEAU et al., 1998; CAMAZINE et al., 2001; FOURCASSIÉ; OLIVEIRA, 2002; THERAULAZ; BONABEAU; DENEUBOURG, 1998; THERAULAZ et al., 2003). Desde os trabalhos de Goldberg (1974), sabe-se que os térmitas podem responder experimentalmente à adaptação ambiental. Criações de colônias de térmitas sulamericanos já foram realizadas com sucesso por Torales, (1979). Para conhecer a interação entre as atividades dos térmitas e o estado estrutural do solo é necessário analisar os impactos da colônia dentro do seu ambiente físico. No entanto, experimentos de campo com esta abordagem são complexos e onerosos. As observações in vitro das funções escavadoras, construção de terraços, da gênese da porosidade ou da formação de microagregados 
são escassas e devem ser comparadas às estruturas provenientes de observações feitas no campo.

Os primeiros ensaios do comportamento da espécie Cornitermes cumulans (Isoptera, Nasutitermitinae) em um ambiente perturbado mostraram o interesse dessa abordagem (BENITO et al., 2008). A espécie C. cumulans é uma das mais estudadas, chegando a ser considerada como uma espécie-chave, "keystone species" (REDFORD, 1984). O objetivo do trabalho foi observar e descrever as feições oriundas da atividade de colônias C. cumulans quando colocadas sob dois tipos de volumes estruturais coletados no solo. Neste experimento foram testados microagregados soltos, ou seja, a estrutura forte granular característica de horizontes "B latossólico", e estruturas densas, provenientes da atividade de uma minhoca tipo "minhocuçu". As estruturas e microestruturas produzidas em condições padronizadas foram comparadas às observações feitas no campo.

\section{Material e Métodos}

O experimento foi conduzido durante nove meses, entre janeiro e outubro de 2008, em casa de vegetação (Embrapa Cerrados, DF). As temperaturas diurnas oscilaram de 28 a $32^{\circ} \mathrm{C}$ e as noturnas se mantiveram em média em $18^{\circ} \mathrm{C}$. Os ninhos em atividade de Cornitermes cumulans (Isoptera, Nasutitermitinae) foram coletados em uma área de pastagem cultivada (Brachiaria brizantha) implantada a 5 anos, em um Latossolo Vermelho Amarelo, descrito anteriormente (REATTO et al., 2007; OLIVEIRA, 2010). Os seis ninhos eram recentes e de diâmetro entre 17 e $24 \mathrm{~cm}$ e altura de $15 \mathrm{~cm}$.

As estruturas do solo utilizadas associadas às colônias provém do mesmo local onde as colônias de térmitas foram coletadas. As estruturas de coprólitos de minhocuçu, minhocas com comprimento geralmente entre 10 e $30 \mathrm{~cm}$ e diâmetro de $\approx 1 \mathrm{~cm}$ ou mais (BROWN; JAMES, 2007), se apresentam no solo como galerias tubulares preenchidas pelas dejeções, elas perduram muito tempo no solo e apresentam massa volumétrica superior ao solo na qual se encontram (OLIVEIRA, 2010). Estas estruturas foram escolhidas por serem densas e fáceis de serem separadas do solo em grandes quantidades. Estas estruturas densas foram coletadas sistematicamente em trincheiras em camadas de 10 em $10 \mathrm{~cm}$ até $1 \mathrm{~m}$ de profundidade; o volume do solo foi peneirado a $5 \mathrm{~mm}$ in situ, e as estruturas separadas e secas ao ar. A estrutura granular forte do horizonte $\mathrm{Bw}_{3}$ vermelho foi coletada a 3 metros de profundidade e seca ao ar. Esta estrutura foi utilizada sem ser peneirada, apenas homogeneizada. As estruturas densas de coprólitos de "minhocuçu" tem diâmetro de 5 a $35 \mathrm{~mm}$ e densidade do solo média (Ds) de $1,4 \mathrm{~g} \mathrm{~cm}^{-3}$ (Tabela 1).

Tabela 1. Principais características físicas e químicas das amostras de estruturas granular (horizonte $\mathrm{Bw}_{3}$ ) e densa (coprólitos) utilizadas nos vasos do experimento.

\begin{tabular}{lcc}
\hline Parâmetro físico-químico & Estrutura granular & Estrutura densa \\
\hline Cor seca (Munsell) & 5 YR5/8 & 10YR 4/4, 10YR 5/6 e 10YR 6/6 \\
Dp $\left(\mathrm{g} \mathrm{cm}^{-3}\right)$ & 2,72 & $\mathrm{Nd}$ \\
$\mathrm{Ds} *\left(\mathrm{~g} \mathrm{~cm}^{-3}\right)$ & $0,91 / 0,02(\mathrm{n}=4)$ & $1,35 / 0,04(\mathrm{n}=6)$ \\
Argila $\left(\mathrm{g} \mathrm{kg}^{-1}\right)$ & 770 & 670 \\
Silte $\left(\mathrm{g} \mathrm{kg}^{-1}\right)$ & 90 & 175 \\
Areia $\left(\mathrm{g} \mathrm{kg}^{-1}\right)$ & 140 & 213 \\
$\mathrm{CO}\left(\mathrm{g} \mathrm{kg}^{-1}\right)$ & 0,02 & 10,30 a 26,28 \\
pH KCl & 6,1 & $\mathrm{nd}^{* *}$ \\
$\mathrm{CTC}\left(\mathrm{Cmol}^{+} \mathrm{kg}^{-1}\right)$ & 1,53 & $\mathrm{Nd}$ \\
\hline
\end{tabular}

Metodologias (EMBRAPA, 1997): Dp = densidade de partícula, picnômetro; Ds = densidade do solo, cilindro no solo e massa volumétrica em água para estrutura densa; Argila, silte e areia, pipeta; CO, carbono orgânico, oxidação pelo bicromato; CTC, extrator $\mathrm{Ca}(\mathrm{CH} 3 \mathrm{COO}) 2$ e $\mathrm{CH} 3 \mathrm{COOH}$ a pH 7,0. *média e erro padrão. * * nd: não determinado. 
O experimento de manutenção das colônias foi montado conforme Benito et al. (2008), com vasos de PVC de 45 litros, $41 \mathrm{~cm}$ de altura, $43 \mathrm{~cm}$ de diâmetro na borda e $29 \mathrm{~cm}$ na base. Em cada vaso foram feitos orifícios laterais de $3 \mathrm{~cm}$ para o encaixe de mangueiras de PVC transparente (Figura 1). A mangueira $B$ liga o vaso A onde se encontra a colônia, mais parte da alimentação, com o vaso C, somente com alimentação.

Figura 1. Esquema de manutenção de colônias de térmitas em condições controladas: a) vaso com solo, ninho e alimento; b) mangueira transparente para conexão entre os vasos; c) vaso para alimentação; 1) ninho de Cornitermes cumulans; 2) tubo de PVC para fornecimento de água; 3) cápsula porosa.

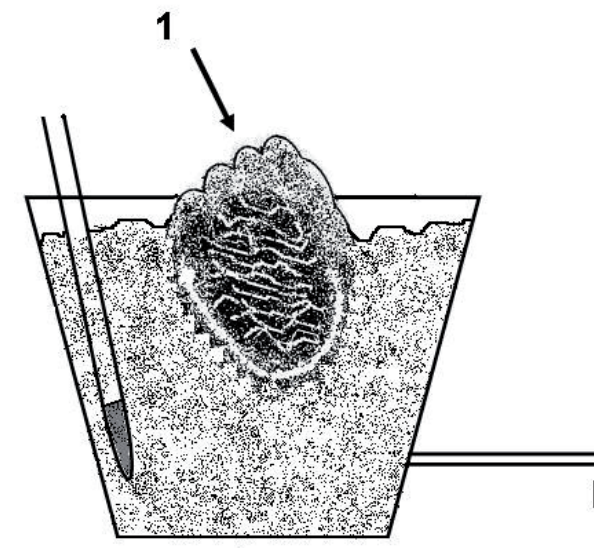

A

Fonte: Elaboração dos autores.

Para cada estrutura de solo testada foram feitas duas repetições (cada repetição constou de um conjunto de dois vasos), sendo utilizada a mesma estrutura no vaso onde foi colocado o ninho e no que foi utilizado somente para alimentação da colônia. Para alimentação das colônias foi fornecido palha de espiga de milho e fezes bovina colocadas na superfície, no vaso A e no vaso C. No final do experimento foram dissecados 4 conjuntos de vasos com material denso, 2 da camada $0-10 \mathrm{~cm}$ e 2 da camada 30-100 cm do solo, e 2 conjuntos de vasos com material granular do horizonte $\mathrm{Bw}_{3}$.

$\mathrm{O}$ fornecimento de água foi realizado por um tubo de PVC de $70 \mathrm{~cm}$ de altura com $5 \mathrm{~cm}$ de diâmetro tendo uma cápsula porosa na extremidade dentro do solo ( $\mathrm{n}^{\circ}$ 653X02B1M3, Soil Moisture Equipament Corp, USA, modelo B01M3, com diâmetro de poro entre 1 a $2 \mu \mathrm{m}$ ) e aberto na extremidade superior. Esse tubo foi introduzido em cada vaso ficando a cápsula porosa entre 2 a $3 \mathrm{~cm}$ do fundo do vaso. A cápsula porosa permite o fornecimento de água sem saturar o solo. A água foi fornecida de maneira a manter o solo na capacidade de campo.

Depois de nove meses os vasos foram dissecados como segue: a cada $5 \mathrm{~cm}$ de espessura foi retirado, cuidadosamente para não destruir as estruturas, uma porção circular do vaso. As estruturas foram descritas e amostradas nos vasos e nas mangueiras para análise micromorfológica e comparação com amostras coletadas no campo.

Os teores de $\mathrm{C}$ total e $\mathrm{N}$ total de coprólitos dos térmitas amostrados nos vasos foram comparados com três tipos de amostras densas oriundas de diferentes camadas do solo, como segue: 1) coprólitos oriundos da camada 0-10 cm (campo); 2) coprólitos da camada 30-100 cm (campo); 3) coprólitos da camada $>100 \mathrm{~cm}$ (campo); 4) coprólitos da camada 0-10 cm retrabalhados por C. cumulans (vaso); 5) coprólitos da camada $>30 \mathrm{~cm}$ retrabalhados por $C$. cumulans (vaso). As determinações de $\mathrm{N}$ total e $\mathrm{C}$ total foram realizadas por oxidação a seco pelo auto- 
analisador CHN (Analyzer CHN Fisons/Carlo Erba NA 2000). Os teores em $\mathrm{C}$ e $\mathrm{N}$ foram comparados pelo teste de Tukey.

\section{Resultado e Discussão}

Modelo experimental com volumes macroestruturais densos (coprólitos de minhocuçu)

As colônias mantiveram as atividades de forrageamento e construção do ninho até o final do experimento. Nos vasos A, que continham os ninhos, a atividade foi intensa para todas as camadas. Fato também observado nos vasos de forrageamento (C). Houve expansão do ninho de C. cumulans (Figura 2a, b, c). Foi observado a construção de um espaço vazio de $10 \mathrm{~mm}$ entre o volume de estruturas densas dentro do vaso e a câmara celulósica (Figura 2d). Esse espaço vazio é a bolsa de manutenção climática refeita pelos térmitas. No campo, esta bolsa é a paraécia (GRASSÉ, 1984), espaço que existe entre o ninho e o solo que o circunda, destinado à manutenção de umidade e temperatura constantes no interior do ninho. As paredes das paraécias nos experimentos, localizadas na base dos ninhos, eram menos rígidas que aquelas observadas no campo, que se individualizam facilmente quando o ninho era dissecado, enquanto na dissecação dos vasos elas eram frágeis e sem rigidez ao tentar separá-las.
Ao retirar o material utilizado para alimentação, nos vasos C, verificou-se que os coprólitos tinham sido parcialmente ou completamente retrabalhados, dando origem a uma estrutura granular forte e fina, composta de excrementos de C. cumulans e restos das estruturas densas (Figura 3). Não importando a profundidade de origem do solo dos coprólitos (vaso A e vaso C), as estruturas densas foram utilizadas pelos térmitas para novas construções. Os coprólitos que ainda restavam estavam cobertos por excremento de térmitas formando pequenos pontos escuros, em alguns casos formando um revestimento de cor preta (Figuras 2d e 3).

A dissecação dos vasos permitiu verificar que os térmitas construíram galerias que circundam o vaso da superfície até fundo em todas as direções, explorando todo o volume disponível. Essas galerias foram construídas entre as macroestruturas densas, tinham paredes frágeis, mas, bem definidas, feitas somente com excremento de cor preta, não sendo observado o adensamento com argila, como no campo. Nenhuma tendência direcional para construção dos túneis foi observada, uma vez que o recurso trófico se encontrava no vaso A e no vaso C, confirmando as observações feitas por Nobre, Nunes e Bignell (2007) que estudaram a geometria dos túneis de Reticulitermes grassei (Rhinotermitidae) em laboratório.

Figura 2. Modelo experimental com volumes macroestruturais densos submetidos à atividade de térmitas Cornitermes cumulans: a) ninho no início do experimento; b) e c) ninhos no final do experimento sob estruturas densas; d) detalhe do espaço vazio entre o solo e a câmara celulósica (vista de cima).

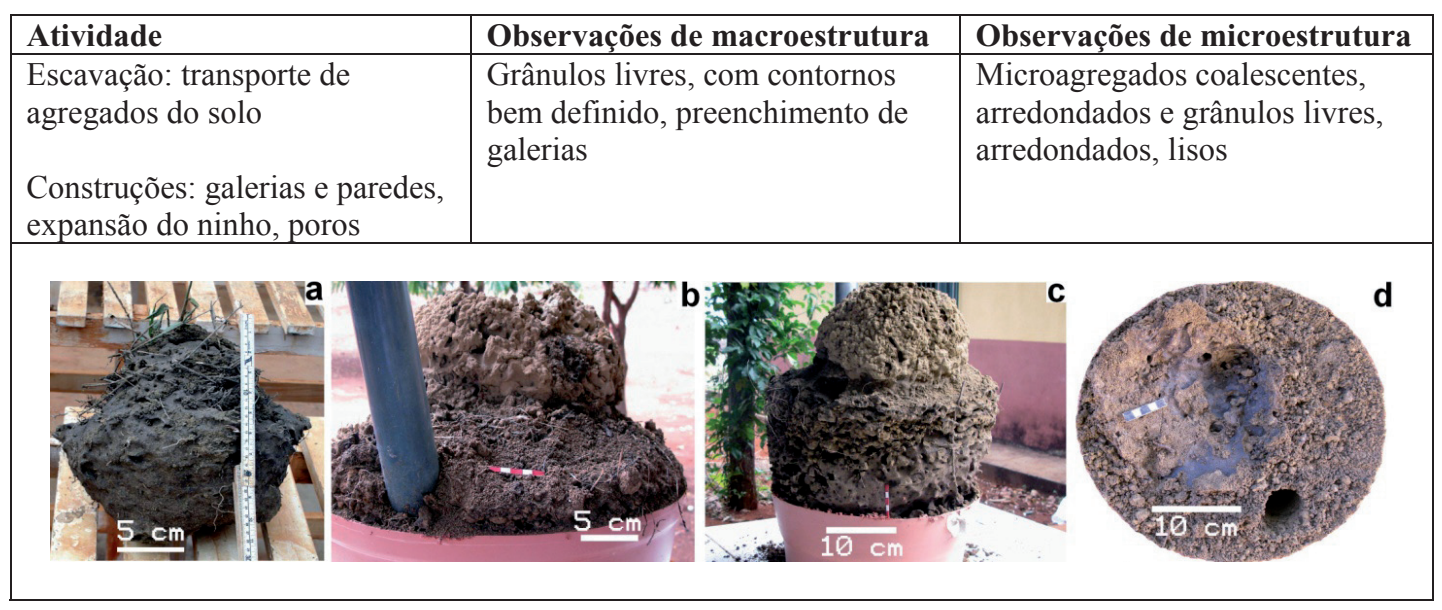

Fonte: Elaboração dos autores. 
Figura 3. Efeito da atividade de Cornitermes cumulans nos volumes macroestruturais densos (coprólitos). a) na parte baixa, as estruturas em estado inicial antes do experimento e, acima, diferentes estados de desmantelamento pelos térmitas; b) restos de estrutura densa e agregados diversos após o término do experimento.
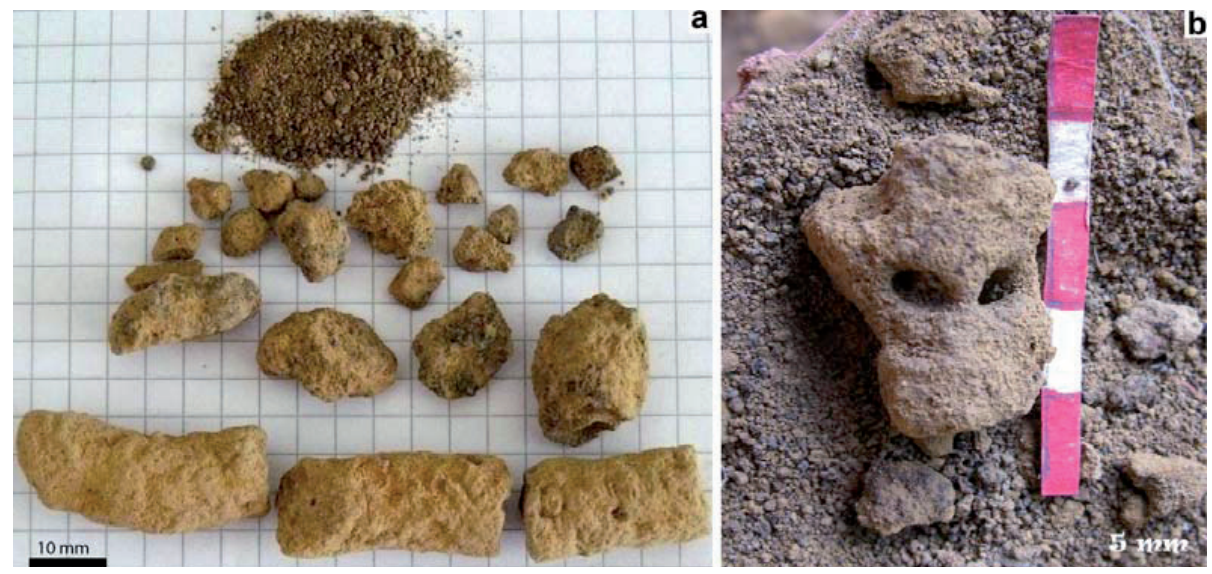

Fonte: Elaboração dos autores.

Modelo experimental com estrutura granular forte (horizonte $\mathrm{Bw}_{3}$ do solo)

Nos vasos com o material do horizonte $\mathrm{Bw}_{3}$ as colônias tiveram atividade reduzida em comparação ao tratamento com estruturas densas. No entanto, observou-se atividade de construção com material retrabalhado na base do ninho, algumas estruturas de paredes eram rígidas, mas as paredes dos alvéolos e galerias eram frágeis, e os agregados pouco coerentes. Em uma das duas repetições deste modelo observou-se a construção de uma galeria dentro da mangueira $\mathrm{B}$. No vaso $\mathrm{A}$, a atividade foi fraca e no vaso $\mathrm{C}$, muito fraca, constando somente do percurso até a alimentação.

Novas construções de galerias e estruturas em todos os vasos

No caso das estruturas densas de "minhocuçu", dentro da mangueira B que liga os dois vasos, foi possível observar a construção de uma galeria, já no primeiro mês do experimento, com parede espessa e resistente, chegando a $10 \mathrm{~mm}$ de espessura (Figura 4). A análise microestrutural mostrou uma trama porfírica aberta, a porosidade destas paredes é nula, apareceram apenas fissuras devido a acomodação orientada. Estas paredes eram compostas de microagregados arredondados, rugosos a lisos formando uma microestrutura granular adensada orientada e bem desenvolvida (Figura 5a), idênticas àquelas identificadas em amostras coletadas no campo (Figura 5b), as paredes continham grãos de quartzo e nódulos de ferro (Figura 5d).

$\mathrm{Na}$ parte basal do ninho os térmitas construíram algumas feições bem rígidas, que permitiram delimitar porções de galerias entre as estruturas densas, onde observou-se canais de preenchimento idênticos aos encontrados nas amostras coletadas no campo (Figura 5c). Este fato foi observado, tanto em vaso com as estruturas densas, quanto em vasos com solo estruturado do horizonte $\mathrm{Bw}_{3}$.

As observações micromorfológicas das galerias construídas nas mangueiras (B) não evidenciaram tendência de preferência por qualquer tipo de partículas do solo pelos térmitas. As partículas do solo foram orientadas em faixas e com microestrutura laminar; microfeições semelhantes também foram relatadas por Lee e Wood (1971) e Sarcinelli et al. (2009), conforme Figura 5a. Esta orientação se dá possivelmente no momento da construção da galeria, quando as partículas de solo, ainda úmidas, são prensadas umas contra as outras, fato semelhante ao 
observado em feições coletadas no campo (Figura 5b). A análise micromorfológica evidenciou que a porosidade nas estruturas produzidas foi quase nula. As estruturas continham grãos de quartzo que mediram de $<0,1$ a $1 \mathrm{~mm}$ (Figura 5d), com microagregados em formato arredondado $(2 \mathrm{~mm})$ formados pela junção de outros microagregados, dando origem a microestruturas adensadas (Figura 5d).

Figura 4. Estruturas produzidas por Cornitermes cumulans no experimento com estruturas densas: a) estrutura produzida na parte inferior do ninho, o circulo indica uma galeria passando ao meio, a seta o acúmulo de matéria orgânica; b) estrutura produzida no interior da mangueira (B), a seta indica a galeria principal para travessia entre vaso A e C.
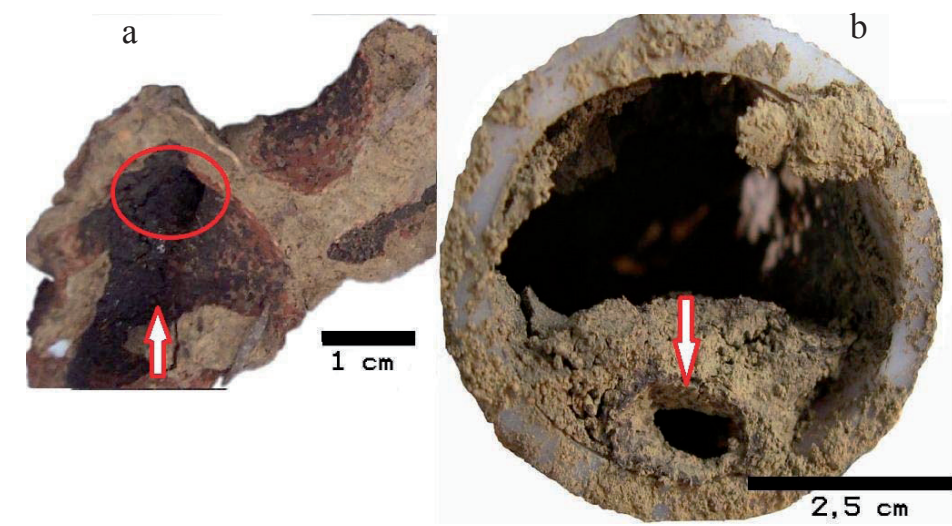

Fonte: Elaboração dos autores.

Figura 5. Fotomicrografias de estruturas produzidas por Cornitermes cumulans: a) o círculo mostra a microestrutura estriada constituída por dejeções, minerais e microagregados do solo (experimento com estrutura granular do horizonte $\mathrm{Bw}_{3}$ ); b) parede de galeria principal lateral, periécia, que sai do ninho em direção a subsuperfície na camada de solo a $45 \mathrm{~cm}$ de profundidade, o círculo mostra a microestrutura estriada constituída por dejeções, minerais e microagregados do solo (proveniente do campo); c) galeria com preenchimento denso, amostra de um volume de material abaixo da colônia (experimento com estrutura granular do horizonte $\mathrm{Bw}_{3}$ ); d) estrutura da parede da galeria no interior da mangueira (B) com fissuras de dessecamento, grãos de quartzos (setas vermelhas) e nódulos de ferro típicos puro (setas brancas).

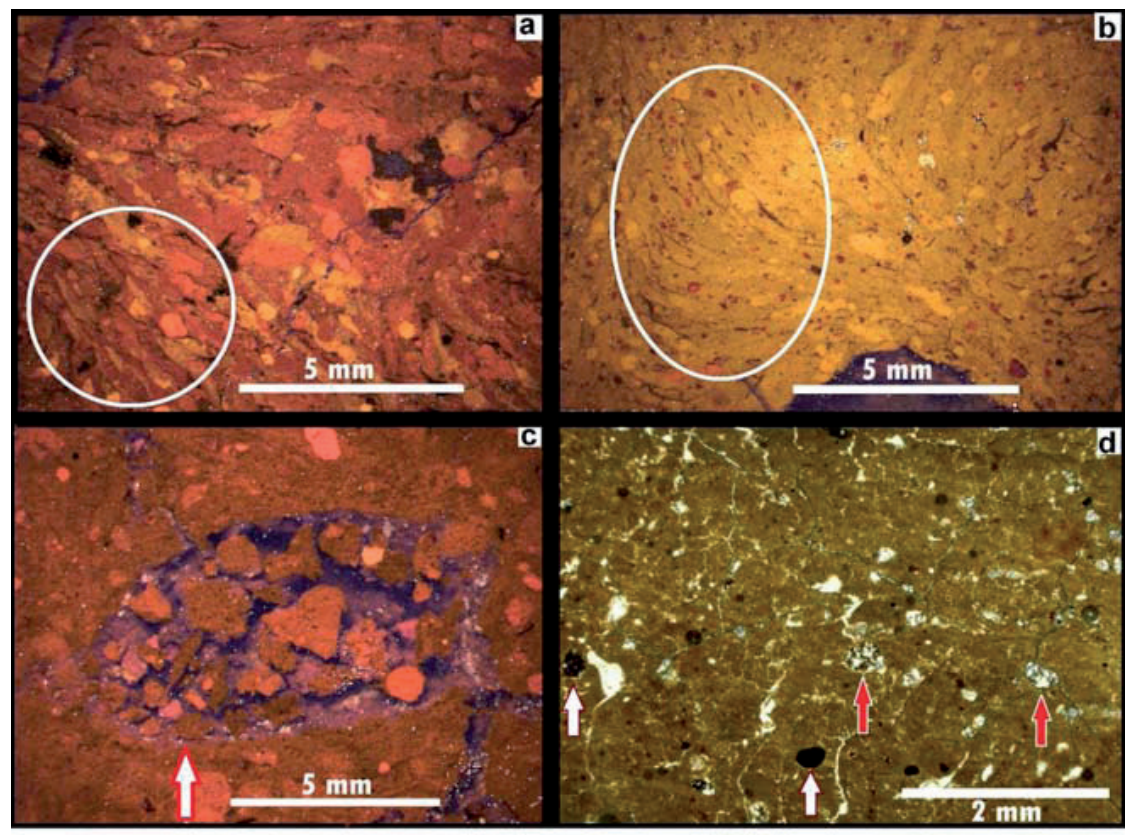

Fonte: Elaboração dos autores. 
Devido à fragilidade das galerias, não foi possível coletar sistematicamente amostras dos experimentos nos vasos para confecção das lâminas delgadas. Era esperado que os térmitas construíssem galerias firmes, no entanto, verificou-se que estes foram oportunistas, utilizando-se dos espaços vazios entre as estruturas densas para se movimentarem (Figura 6b). As galerias observadas eram extremamente frágeis, porém, bem definidas (Figura 6a), sem uma parede adensada com argila, como aquelas encontradas no campo (Figura 6c). No experimento de casa de vegetação, essas galerias foram formadas de um revestimento escuro com $2 \mathrm{~mm}$ de espessura e diâmetro entre 1 a $2 \mathrm{~cm}$.

Em condições de laboratório, Arab e CostaLeonardo (2005) verificaram estreita relação entre umidade e temperatura com a área escavada por Coptotermes gestroi e Heterotermes tenuis (Rhinotermitidae). Lee, $\mathrm{Su}$ e Lee (2009) demonstraram que os térmitas podem restringir o número de túneis que constroem para aumentar a eficiência de transporte de alimento. Nesse estudo, onde a temperatura e umidade foram as mesmas para todos os ninhos da espécie de C. cumulans, as atividades de retrabalhar o solo e construir galerias foram intensas, sugerindo que o padrão de escavação é mais um fator comportamental, podendo estar ou não associado à busca por alimento. Para as colônias associadas às estruturas densas, as atividades se estenderam para o vaso $\mathrm{C}$, dessa forma, a atividade de construção se deu concomitante ao forrageamento, ocorrendo nos vasos A e C.Em ambiente natural os térmitas constroem galerias transversais, horizontais e verticais que podem atingir grandes profundidades (LEPAGE; MOREL; RESPLENDINO, 1974), em busca de água e material argiloso úmido (LEPRUN, 1976). Confirmando as descrições na África, as observações in situ, onde as colônias do C. cumulans foram coletadas, mostraram galerias verticais desenvolvidas até 3 a 4 metros de profundidade (OLIVEIRA, 2010).

A análise da relação das colônias de C. cumulans segundo o tipo de volume estrutural ofertado neste experimento é difícil de interpretar. Para o material do horizonte $\mathrm{Bw}_{3}$, as estruturas que foram produzidas são pouco relevantes; por outro lado, os térmitas tiveram um impacto morfológico quando as colônias estavam associadas as macroestruturas densas. Com os volumes granulares, ou seja, microagregados justapostos sem coesão, a atividade dos térmitas produziu estruturas específicas como as galerias que permitiram atingir o recurso alimentar, assim como o remanejamento da base do ninho. Não se pode considerar que a localização da oferta trófica pode ter sido um fator para restrição das escavações no caso do $\mathrm{Bw}_{3}$, pois as condições foram as mesmas para os dois tipos de estruturas colocadas com as colônias. Uma possível interação com fatores bioquímicos do material do horizonte $\mathrm{Bw}_{3}$ pode ter ocorrido, não se tem relato bibliográfico sobre o tema. No entanto, a hipótese mais coerente seria de considerar que o material denso tinha que ser desestruturado para que as colônias pudessem se desenvolver, no caso do material granular forte do horizonte $\mathrm{Bw}_{3}$, os agregados livres permitiram o mínimo de construção necessária para manter as colônias ativas. Finalmente, as macroestruturas densas foram retrabalhadas, alteradas, as colônias de térmitas produziram e isolaram assim agregados livres e grânulos. Em particular, a estruturação da base dos ninhos levaram a um padrão de microagregação que já havia sido observado no campo.

Do ponto de vista metodológico, este tipo de experimento deve ser repetido para normalizar a análise de novas estruturas produzidas e, em particular, estudar os padrões de distribuição dos materiais no plasma, definir se as microestruturas são aproveitadas e/ou refeitas, e efetuar uma análise mais consistente da macro e microporosidade criadas. 
Figura 6. Galerias de térmita Cornitermes cumulans: a) galeria em vaso experimental com macroestrutura densa, seta mostra revestimento escuro na parede com depósito de matéria orgânica; b) em vaso experimental, galerias entre as estruturas densas, parcialmente alteradas e forradas com agregados, seta mostra caminho percorrido pelos térmitas; c) galeria no campo, seta indica a parede da galeria bem definida e acúmulo de matéria orgânica.
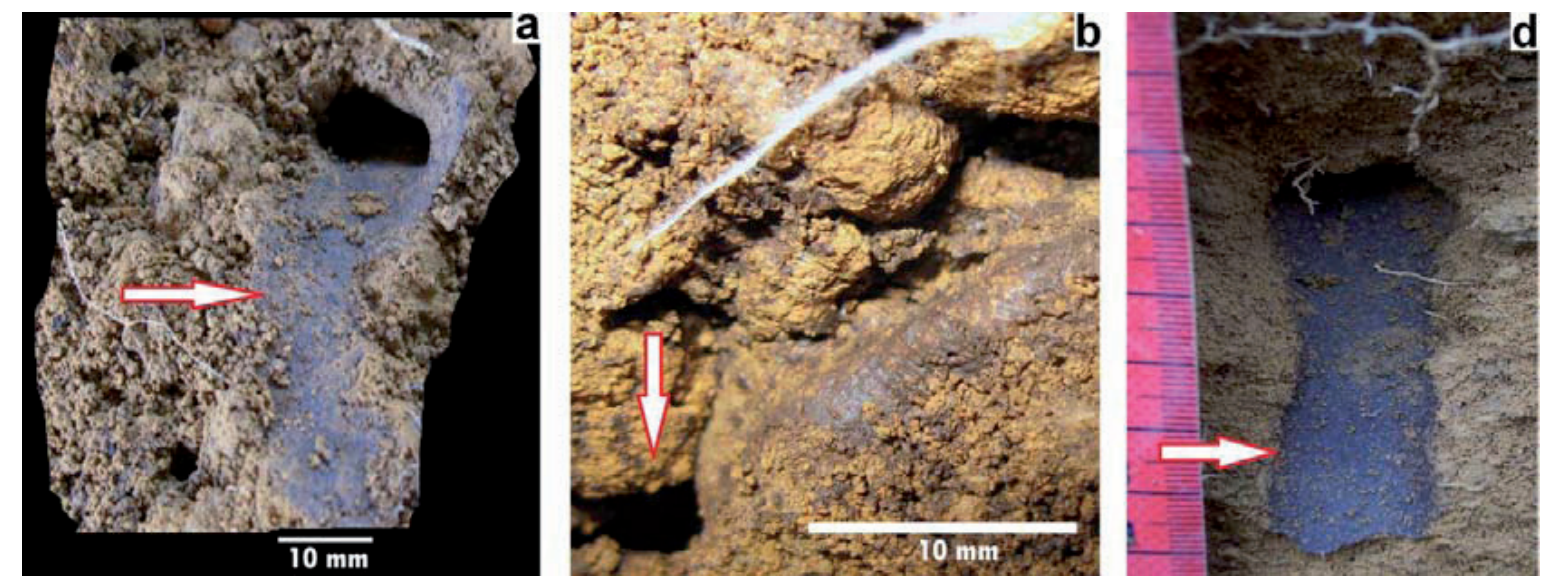

Fonte: Elaboração dos autores.

Carbono e Nitrogênio das Macroestruturas Densas

Observou-se que a atividade dos C. cumulans nos vasos conduziu à uma produção de agregados oriundos das macroestruturas densas (Figura 2). No início do experimento as macroestruturas densas formadas pelos coprólitos amostrados no campo, apresentaram uma concentração de carbono (C) de $25,02 \mathrm{~g} . \mathrm{kg}^{-1}$, quando amostrados no horizonte 0-10 cm do solo, e valores de 9,6 g. $\mathrm{kg}^{-1}$, quando amostrados em horizontes mais profundos (Tabela 2). Os valores de nitrogênio $(\mathrm{N})$ seguiram o mesmo padrão em função da profundidade de amostragens. Em diversos locais dos vasos foram feitas amostragens para se obter uma amostra composta de material retrabalhado pelos térmitas. Nos vasos do experimento os valores de $\mathrm{C}$ não foram significativamente alterados para o material oriundo do horizonte $0-10 \mathrm{~cm}$ do solo, isto se explica pelo valor já elevado de $\mathrm{C}$ e $\mathrm{N}$ do material inicial. No entanto, quando se observa o material denso dos horizontes de 30 a $100 \mathrm{~cm}$ de profundidade, o valor de C aumentou de 9,69 $\mathrm{g} \mathrm{kg}^{-1}$ para 24,19 $\mathrm{g} \mathrm{kg}^{-1}$, valores semelhantes àqueles observados nos materiais do horizonte $0-10 \mathrm{~cm}$, o aumento de $\mathrm{N}$ foi proporcional. Dessa forma, em nove meses de experimento, a redistribuição de matéria orgânica parece ser eficaz. Este resultado é coerente com a síntese de Holt e Lepage (2000) que relatam o enriquecimento de C e $\mathrm{N}$ em ninhos de térmitas, em comparação com o solo adjacente. A origem destes materiais orgânicos é ligada às dejeções que foram depositadas em fase liquida e, posteriormente endurecidas durante a secagem, semelhantes observações foram feitas no campo (Figura 6c; OLIVEIRA, 2010) e relatadas nas observações de Grassé (1984) nos ninhos e galerias de C. cumulans do estado de São Paulo. 
Tabela 2. Teores totais de carbono e nitrogênio das estruturas densas (coprólitos) amostradas no campo em três profundidades do solo e depois da ação do Cornitermes cumulans nos vasos.

\section{Estruturas}

(1) coprólitos oriundos da camada $0-10 \mathrm{~cm}$ (campo)

(2) coprólitos da camada $30-100 \mathrm{~cm}$ (campo)

(3) coprólitos da camada $>100 \mathrm{~cm}$ (campo)

(4) coprólitos da camada $0-10 \mathrm{~cm}$ (1) retrabalhados por C. cumulans (vaso)

(5) coprólitos da camada 30-100 cm (2) retrabalhados por C. cumulans (vaso)

\begin{tabular}{ccc} 
C total* & N total* & C/N \\
\cline { 1 - 2 } $25,02^{\text {a }}$ g.kg $^{-1}$ & $1,33^{\mathrm{b}}$ & 18,81 \\
$9,69^{\mathrm{b}}$ & $0,60^{\mathrm{c}}$ & 16,12 \\
$9,66^{\mathrm{b}}$ & $0,58^{\mathrm{c}}$ & 16,52 \\
$25,15^{\mathrm{a}}$ & $1,42^{\mathrm{a}}$ & 17,71 \\
$24,19^{\mathrm{a}}$ & $1,27^{\mathrm{b}}$ & 19,02 \\
\cline { 1 - 2 } & &
\end{tabular}

* Resultados com letras diferentes na mesma coluna são significativamente diferentes pelo teste Tukey, $p \geq 0,95$.

Fonte: Elaboração dos autores.

\section{Conclusões}

As colônias de Cornitermes cumulans exploram o ambiente de forma aleatória em todas as direções, retrabalham estruturas densas e reagregam as partículas do solo para construção de galerias.

Todas as estruturas densas ofertadas às colônias foram parcialmente ou completamente modificadas, aumentando as concentrações de matéria orgânica das estruturas densas oriundas de horizontes profundos do solo.

As atividades de Cornitermes cumulans foram menos intensas sobre o material do horizonte $\mathrm{Bw}_{3}$.

\section{Agradecimentos}

CAPES (Brasil) projeto CAPES/COFECUB N ${ }^{\circ}$ 633/09 "Caracterização das atividades coletivas de forrageamento e construção de ninhos de formigas e térmitas neotropicais e estudo de seu impacto no ambiente". O trabalho foi desenvolvido no âmbito da parceria EMBRAPA Cerrados-IRD, projeto ESSAIM [Estado estrutural de solos e atividade de insetos sociais em ambientes tropicais: análise de uma interação modelo térmita-solo] (Programa ANR-INSU-PNBC, França).

\section{Referências}

ARAB, A.; COSTA-LEONARDO, A. M. Effect of biotic and abiotic factors on the tunneling behavior of Coptotermes gestroi and Heterotermes tenuis (Isoptera: Rhinotermitidae). Behavioural Processes, 70, p. 32-40, 2005.

BENITO, N. P.; SOARES, R. S.; CAMARGO, A. J. A. de; BROSSARD, M. Criação de colônias de cupins de montículos em casa de vegetação. CONGRESSO BRASILEIRO DE ENTOMOLOGIA, 22., 2008, Uberlândia. Anais... Uberlândia: Sociedade Brasileira de Entomologia, 2008. <http://www.seb.org.br/eventos/ CBE/XXIICBE/resumos/R0290-4.html.>. Acesso em: 01 dez. 2011.

BIGNELL, D. E. Termites as soil engineers and soil processors. In: KONIG, H.; VARMA, A. (Ed.). Soil Biology. Intestinal microorganisms of soil invertebrates. Berlin, Heidelberg: Springer-Verlag, 2006. v. 6, p. 183220.

BONABEAU, E.; THERAULAZ, G.; DENEUBOURG, J. L.; FRANKS, N.; RAFELSBERGER, O.; JOLY, J. L.; BLANCO, S. A model for the emergence of pillars, walls and royal chamber in termite nests. Philosophical Transactions of the Royal Society of London Serie B Biological Sciences, London, v. 353, n. 1373, p. 15611576, 1998.

BROSSARD, M.; LÓPEZ-HERNÁNDEZ, D.; LEPAGE, M.; LEPRUN, J.-C. Nutrient storage in soils and nests of mound-building Trinervitermes termites in Central Burkina Faso: consequences for soil fertility. Biology and Fertility Soils, Heidelberg, v. 43, n. 4, p. 437-447, 2007. 
BROWN, G. G.; JAMES, S. W. Ecologia, biodiversidade e biogeografia das minhocas no Brasil. In: BROWN, G. G.; FRAGOSO, C. (Ed.). Minhocas na América Latina: biodiversidade e ecologia. Londrina: Embrapa Soja, 2007. p. 297-381.

CAMAZINE, S.; DENEUBOURG, J. L.; FRANKS, N.; SNEYD, J.; THERAULAZ, G.; BONABEAU, E. Selforganization in biological systems. Princeton: Princeton University Press, 2001.

CHAPUIS-LARDY, L.; LE BAYON, R. C.; BROSSARD, M.; LÓPEZ-HERNÁNDEZ, D.; BLANCHART, E. Role of soil macrofauna in P cycling. In: BÜNEMANN, E. K.; OBERSON, A.; FROSSARD, E. (Ed.). Phosphorus in Action - Biological Processes in Soil Phosphorus Cycling. NY, USA: Springer, 2011. p. 199-213. (Springer Soil Biology Series, 26).

DEBRUYN, L. A. L.; CONACHER, A. J. Soil modification by termites in the central wheat-belt of Western-Australia. Australian Journal of Soil Research, Australia, v. 33, n. 1, p. 179-193, 1995.

DECÄENS, T.; LAVELLE, P.; JIMÉNEZ, J. J.; ESCOBAR, G.; RIPPSTEIN, G.; SCHNEIDMADL, J.; SANZ, J. I.; HOYOS, P.; THOMAS, R. J. Impacto del uso de la tierra en la macrofauna del suelo de los llanos orientales de Colombia. In: JIMÉNEZ, J. J.; THOMAS, R. J. (Ed.). El arado natural: las comunidades de macroinvertebrados del suelo en las savanas neotropicales de Colombia. Cali, Colombia: Centro Internacional de Agricultura Tropical, 2003. p. 21-45. (Publicación CIAT, 336).

EMPRESA BRASILEIRA DE PESQUISA AGROPECUÁRIA - EMBRAPA. Manual de métodos de análise de solos. 2. ed. Rio de Janeiro: Empresa Brasileira de Pesquisa Agropecuária, 1997.

FERREIRA, R. R. M.; TAVARES FILHO, J.; FERREIRA, V. M. Efeitos de sistemas de manejo de pastagens nas propriedades físicas do solo. Semina: Ciências Agrárias, Londrina, v. 31, n. 4, p. 913-932, 2010.

FOURCASSIÉ, V.; OLIVIERA, P. Foraging ecology of the giant ant Dinoponera gigantea (Hymenoptera, Formicidae, Ponerinae): activity schedule, diet, and spatial foraging patterns. Journal of Natural History, London, v. 36, n. 18, p. 2211-2227, 2002.

GARNIER-SILlAM, E.; TOUTAIN, F.; RENOUX, J. Comparaison de l'influence de deux termitières (humivore et champignonniste) sur la stabilité structurale des sols forestiers tropicaux. Pedobiologia, Jena, Alemanha, v. 32, n. 1-2, p. 89-97, 1988.
GOLDBERG, J. La construction chez le termite de Saintonge. L'adaptation des galeries tunnels à la topographie et le tracé minimal. Comptes Rendus Académie des Sciences Paris, Sér. D, Paris, v. 278, n. 24, p. 3127-3130, 1974.

GRASSÉ, P. P. La reconstruction du nid et les coordinations interindividuelles chez Bellicositermes natalensis et Cubitermes sp. La théorie de la stigmergie : essais d'interprétation du comportement des termites constructeurs. Insectes Sociaux, Masson, v. 6, n. 1, p. 41-84, 1959.

Termitologia. Tome 2. Fondation des sociétés Construction. Paris: Masson, 1984.

HOLT, J. A.; LEPAGE, M. Termites and Soil Properties. In: ABE, T.; BIGNELL, D. E.; HIGASHI, M. (Ed.). Termites, evolution, sociality, symbiosis, ecology. Dordrecht: Kluwer Academic, p. 389-407, 2000.

LAVELLE, P. Faunal activities and soil processes: adaptive strategies that determine ecosystem function. In: BEGON, M.; FITTER, A. (Ed.). Advances in ecological research. London, San Diego: Academic Press, v. 27, 1997. p. 93-132.

LAVELlE, P.; SPAIN, A. V. Soil ecology. Dordrecht: Kluwer Academic Publishers, 2001.

LEE, S.-H.; SU, N.-Y.; LEE, M. Why is the number of primary tunnels of the formosan subterranean termite, Coptotermes formosanus Shiraki (Isoptera: Rhinotermidae), restricted during foraging. Journal of Asia-Pacific Entomology, v. 12, n. 3, p. 151-154, 2009.

LEE, K.; WOOD, T. Termites and soils. London: Academic Press, 1971.

LEPAGE, M.; MOREL, G.; RESPLENDINO, C. Découverte de galeries de termites atteignant la nappe phréatique profonde dans le Nord du Sénégal. Comptes Rendus Académie des Sciences Paris, Sér. D, Paris, t. 278, p. 1855-1858, 1974.

LEPRUN, J-C. Une construction originale hypogée pour le stockage de l'eau par les termites en régions sahelosoudaniennes de Haute-Volta. Pedobiologia, Jena, v. 16, n. 8 , p. 451-456, 1976 .

LÓPEZ-HERNÁNDEZ, D. Nutrient dynamics (C, N and $\mathrm{P}$ ) in termite mounds of Nasutitermes ephratae from savannas of the Orinoco Llanos (Venezuela). Soil Biology and Biochemistry, Oxford, v. 33, n. 6, p. 747-753, 2001.

LÓPEZ-HERNÁNDEZ, D.; BROSSARD, M.; FARDEAU, J-C.; LEPAGE, M. Effect of different termite feeding groups on $\mathrm{P}$ sorption and $\mathrm{P}$ availability in African and south American savannas. Biology and Fertility of Soils, Germany, v. 42, n. 3, p. 207-214, 2006. 
NOBRE, T.; NUNES, L.; BIGNELL, D. Tunnel geometry of the subterranean termite Reticulitermes grasse (Isoptera: Rhinotermitidae) in response to sand bulk density and the presence of food. Insect Science, New York, v. 14, n. 6, p. 511-518, 2007.

OLIVEIRA, M. I. L. de. Bioestruturas em um Latossolo Vermelho Amarelo produzidas por organismos geófagos. 2010. Tese (Doutorado em Agronomia) - Universidade Estadual de Londrina, Londrina.

OLIVEIRA, M. I. L.; BRUNET, D.; MITJA, D.; CARDOSO, W. S.; BENITO, N. P.; GUIMARÃES, M. F.; BROSSARD, M. Incidence of epigeal nestbuilding termites in brachiaria pastures in the Cerrado. Acta Scientiarum. Agronomy, Maringá, v. 33, n. 1, p. 181-185, 2011.

REATTO, A.; BRUAND, A.; SILVA, E. M.; MARTINS, E. S.; BROSSARD, M. Hydraulic properties of the diagnostic horizon of Latosols of a regional toposequence across the Brazilian Central Plateau. Geoderma, Amsterdam, v. 139, n. 1-2, p. 51-59, 2007.

REDFORD, K. The termitaria of Cornitermes cumulans (Isoptera, Termitidae) and their role in determining a potential keystone species. Biotropica, Washington, US, v. 16, n. 2, p. 112-119, 1984.
SARCINELLI, T. S.; SCHAEFER, C. E. G. R.; LYNCH, L. S.; ARATO, H. D.; VIANA, J. H. M.; ALBUQUERQUE FILHO, M. R.; GONÇALVES, T. T. Chemical, physical and micromorphological roperties of termite mounds and adjacent soils along a toposequence in Zona da Mata, Minas Gerais State, Brazil. Catena, v. 76, n. 2, p. 107-113, 2009.

THERAULAZ, G.; BONABEAU, E.; DENEUBOURG, J. L. The origin of nest complexity in social insects. Complexity, v. 3, n. 6, p. 15-25, 1998.

THERAULAZ, G.; GAUTRAIS, J.; CAMAZINE, S.; DENEUBOURG, J. L. The formation of spatial patterns in social insects: from simple behaviours to complex structures. Philosophical Transactions of the Royal Society of London, London, Serie A, v. 361, p. 12631282, 2003.

TORALES, G. J. Contribución al conocimiento de los termitas de Argentina (Pcia. de Corrientes). Nasutitermes fulviceps (Isoptera: Termitidae). I. Comportamiento constructor en condiciones de laboratorio. Facena, Corrientes, Argentina, v. 3, p. 119-133, 1979. 